\title{
Designing Digital Mindfulness-based Interventions for Older Informal Carers
}

\author{
Michael Wilson \\ NetwellCASALA \\ Dundalk Institute of Technology \\ Dundalk, Ireland \\ michael.wilson@dkit.ie
}

\author{
Julie Doyle \\ NetwellCASALA \\ Dundalk Institute of Technology \\ Dundalk, Ireland \\ julie.doyle@casala.ie
}

\author{
Gerry McTaggart \\ School of Health and Science \\ Dundalk Institute of Technology \\ Dundalk, Ireland \\ gerry.mctaggart@dkit.ie
}

\begin{abstract}
Stress is prevalent among older informal carers and has been shown to lead to negative developments in physical and psychological health, as well as overall wellbeing. One area of wellbeing that can be impacted is sleep, with high stress levels leading to poor sleep and poor sleep causing stress. Mindfulness-based interventions (MBIs) have grown in popularity in recent years and have been shown to reduce stress and increase quality of life among older informal carers. MBls delivered digitally have the potential to be used by and be of benefit to a wide group of people. Based on the apparent gaps in the literature concerning the area of mobile device/computer-mediated MBIs for older informal carers, further research is needed in this area. An opportunity also lies in the use of wearable activity trackers to monitor any potential improvements in sleep, due to reduced stress. This paper focuses on the design of a digital application delivering MBIs, to support older informal carers to manage stress and sleep. We describe a qualitative study with older informal carers and the resulting digital application while outlining how this system will be trialled with 15 older informal carers over a 12-week period with a view to reducing stress and improving overall wellbeing.
\end{abstract}

Older Adults. Informal Carers. Technology Design. Stress Management. Mindfulness-based Interventions.

\section{INTRODUCTION}

Across the world we are experiencing an increase in the number of older adults, as medical advances, improved diet, and better healthcare have resulted in increased life expectancies. This increase will impact on health services, as it is expected there will be a shortage of healthcare workers to care for the ageing population. It is therefore likely that the burden of care will thus fall on the older person self-managing with support from their informal carer(s) (Consolvo et al. 2009). A census carried out in Ireland in 2011 revealed that $4.1 \%$ of the total population was providing unpaid assistance to others in April 2011. The number of carers aged $60-74$ years old increased by $33.6 \%$ from 2006 to 2011 , with further $39.5 \%$ in the group aged 75 and over (Bardram et al. 2013). The economic impact has been estimated to be in the region of $€ 4$ billion per year. This estimation shows a significant increase from the 2006 estimate of $€ 2.5$ billion.

It is already common for older care recipients to be cared for at home by a family member or friend, who is very often also an older adult. Informal care is care that is provided to a person with whom the informal carer has a personal relationship or close ties (Bulmer et al. 1992, Vaseliou et al, 2017). This care is typically unpaid and involves provision from a few hours a week up to round-the-clock care with co-habitation (Carers UK, 2015). Data from the Irish Longitudinal Study on Ageing (TILDA) shows that the majority of carers (89\%) providing care to community-dwelling older adults are unpaid (Kamiya et al. 2012). There is a large amount of research linking informal caregiving to high levels of burden, anxiety, stress and poor overall wellbeing (Brummett et al. 2006, Bulmer et al. 1992, Care Alliance 2012). Carers of people with dementia (PwD), for example, frequently report feelings of social isolation and inadequate social support, and risk of anxiety and depressive disorders is increased. Contextual factors are also important here; older carers' physical health and mortality can be affected by stress and anxiety, which can in turn compromise ability to care (Brummett et al. 2006). As older adults have lower physiological reserves, they are more likely to be affected by stress (Bulmer et al. 1992, Chen et al. 2013). Carers are seen as a critical health care resource. Evidence on the health effects of caregiving gathered over the last 20 years has helped convince policymakers that this area is a major public health issue (Care Alliance 2012).

As such, it is important to address this issue with a view to exploring means of reducing stress among this cohort. One such approach is through the use of digital MBls and health self-monitoring. This paper provides an insight into the area of stress among informal carers and aims to make a contribution to the field of $\mathrm{HCl}$ by providing an overview of the design of a digital application delivering MBIs. This application and its design has 
been informed by a review of the literature as a well as findings from interviews and focus groups carried out over a two year period with older adults and informal carers.

\section{BACKGROUND AND RELATED WORK}

\subsection{Stress and its impact on caring}

Stress is prevalent among older informal carers. Caregiving can be extremely stressful due not only to the caregiving itself but also to the need to balance work, family and other elements of one's personal life (Chen et al. 2013) As well as objective burdens such as the physical activities associated with caring, there also exist considerable subjective burdens such as emotional distress which arises as a reaction to the caregiving process (Montgomery \& Kosloski 2009, Vaseliou et al. 2017). Chen et al. (2013) point out that caring for older adults is often highly demanding, leading to frustration and sometimes depression in the caregiver. Findings from a study addressing caregivers' experiences revealed feelings of stress, frustration and difficulty across all interview respondents. Vaseliou et al. (2017) present findings from a study exploring informal carers' reflections on experiences of loneliness associated with the caregiving process. Results suggest that the restrictions imposed by the caregiving role can lead to an environment of shrunken personal space and curtailed social interaction. Sentiments of powerlessness, helplessness, and a sense of sole responsibility were also expressed.

Stress has been shown to lead to negative developments in physical and psychological health, as well as overall wellbeing (Adams et al. 2014, Bohlmeijer et al. 2010, Vaseliou et al. 2017). One area of wellbeing that can be impacted is sleep, with high stress levels leading to poor sleep and poor sleep adding to stress (Bohlmeijer et al. 2010). Older adults who have reported difficulties sleeping also report decreased quality of life and higher rates of depression (Bauer et al. 2012). Discussing the area of poor sleep hygiene, Choe et al. (2011) see stress and anxiety, among other things, as key contributors to sleep disturbances. Moreover, there is a tendency for sleep disturbances in older adults to go unreported, which could have a knock on effect on daytime impairment, depression, anxiety, stress, and chronic insomnia (Black et al. 2015).

\subsection{Mindfulness as an intervention}

Mindfulness-based interventions have grown in popularity in recent years and have been shown to reduce stress and increase quality of life among older informal carers (Chiesa \& Serretti 2010, Bohlmeijer et al. 2010). Mindfulness-based interventions (MBIs) are comprised of evidencebased programs that address stress-related ailments through training participants in 'the systematic practice of attending to moment-bymoment experiences, thoughts, and emotions from a non-judgmental perspective'. It is believed to work through arousal and neurocognitive processes that moderate the relationship between perception of stimuli and appraisal (Black et al 2015, Dharmawardene et al. 2016). Mindfulnessbased interventions have been shown to improve health measures, such as anxiety, sleep disturbances, and stress (Wahbeh et al. 2016). Mindfulness itself has been described as an active process during which attention to the present moment is accomplished to allow for a complete, meaningful experiences of what is happening currently, 'without avoiding, judging or ruminating about certain features' (Fresco et al. 2010).

Fiocco et al. (2015) present the findings of a study investigating the effectiveness of a standard 8week $\mathrm{MBI}$ addressing stress on cognition and overall wellbeing in 97 healthy individuals over the age of 60 . Qualitative data from the study suggests that participants who engaged in mindfulnessbased practice felt improved physical and mental awareness, increased patience and acceptance, reduced physical pain and ability to better deal with stressors.

Mindfulness-driven behavior change has also been discussed in the literature. Acceptance of current thoughts and emotions without judgment can allow one to change their behavioral response to current events (Dutton 2008). Indeed, owing to thoughts not necessarily being factual or accurate, a behavioral response may not be required at all (Kristeller et al. 2006). Learning to accept unpleasant thoughts and emotions leads to a realization that a reaction to such thoughts is not required. By encouraging increased self-awareness and self-acceptance, mindfulness enables the individual to concentrate on his/her circumstances and attitudes as opposed to focusing on external forces and influences. This allows in turn for a greater sense of control over goals and behaviours (Dutton 2008). Salmoriago-Blotcher et al. (2013) set out to examine the association between mindfulness training and changes in health-related behaviours. Following an 8-week $\mathrm{MBI}$ addressing stress, participants reported significant improvements in behaviours related to diet and sleep quality. In this instance, mindfulness training was seen to promote favorable changes in healthrelated behaviours, with further research in this area recommended.

Research has demonstrated that an online mindfulness intervention can be effective. The feasibility of an online mindfulness meditation intervention for community-dwelling older 
caregivers has been tested in order to explore its impact on quality of life, caregiver burden, and psychological wellbeing (Tkatch et al. 2017). A total of 40 caregivers were recruited from two community center support groups to participate in an 8-week online mindfulness intervention. Eight modules were delivered twice weekly online, employing both phone and web-interface. The online learning platform contained session materials, downloadable brief meditation practices, access to short learning videos, and other support tools. Participants were free to choose which session to join during the week. As well as this, three of the modules were delivered in-person if participants were able to attend and favored this approach. Results from this study indicate that the intervention reduced caregiver burden, perceived stress, anxiety, and loneliness and improved mental wellbeing. Moreover, this intervention showed that consistent with the literature, the use of technology is a viable intervention for older adults. As such, the online availability of an intervention provides an opportunity for older adults who possibly do not have access to transport or who may not be able to leave the care recipient.

However, there are gaps in the literature concerning the area of mobile device/computermediated stress reduction interventions for older informal carers using MBls. As such, further research is needed in this area. Moreover, the focus in many prior studies has been on designing patient-centric technologies while neglecting to an extent the caregiver and the burdens that can affect health and wellness (Chen et al. 2015).

\subsection{Designing for Older Adults}

A number of design considerations need to be taken into account when designing health and wellbeing applications for older adults (Correira de Barros et al. 2014). While involving users in the design process and following a user-centred design (UCD) approach can address certain issues, other considerations can sometimes be overlooked, such as motivation, engagement, and adherence; and also assumptions pertaining to digital literacy in general (Wahbeh et al. 2016). Indeed, it has been suggested that a 'user-sensitive inclusive design' may be more suitable for less homogenous groups such as older adults, and could address possible drawbacks to some user-centred design techniques. As such, adaptations may need to be made to traditional UCD methods used in Human Computer Interaction $(\mathrm{HCl})$ when working with older adults (Gregor et al. 2002). $\mathrm{HCl}$ research involving older adults is unique in that attention must be paid to both the design, and also to the process; certain issues must be considered, such as preparing sessions, being aware of participants' wellbeing in terms of tiredness and fatigue, and dealing with idiosyncrasies of the age group (Rubin and Chisnell 2008).

\section{METHODOLOGY}

In this paper, we describe a requirements gathering qualitative study that took place over a 2-year period. A total of 20 older adults took part. Requirements gathering consisted of focus groups, and interviews with older adults and informal carers. These are described further below.

\subsection{Requirements Gathering}

A semi-structured focus group lasting 90 minutes was carried out with five older adults, $3 F$, age range 68-86. The participants for this focus group were not informal carers as the initial focus for the research project was on older adults in general. However, as the project advanced and further literature was reviewed, it became apparent that older informal carers could potentially benefit greatly from a health self-management application that provided interventions to help manage stress and improve sleep.

The focus group was divided into 2 sections. The first part explored attitudes to monitoring health and wellness, including stress and sleep, including for example what concerns older adults may have, how they are currently dealt with, how they feel about self-management of these either now or in the future. The second part focused on how participants interpreted data visualizations (presented in the form of paper-based mock-ups) in order to gauge opinion on the most effective means of presenting information through visualizations e.g. using metaphors, bar charts, pie charts etc. to understand how older adults might interpret feedback on their wellbeing. This is to ensure that the feedback in the application is intuitive, easy to interpret and therefore more likely to be effective. The focus group was audio recorded and transcribed to allow for thematic analysis.

A second semi-structured focus group lasting 90 minutes was carried out with four older adults, $2 \mathrm{~F}$, $2 \mathrm{M}$, age range $65-86$. The focus group was divided into 2 sections. The first assessed parameters of health and wellbeing that are considered most important to people. The goal here was to follow up on themes which emerged from the initial focus group so as to gain further insights and to explore whether there was support for opinions that had been put forward by previous participants. The second part focused on participants' opinions on wearable sensors and the idea of continuous tracking, in particular their opinions on the look and feel of two devices (Withings Activite Pop ${ }^{1}$ and

\footnotetext{
${ }^{1}$ Now known as Nokia Health Steel
} 
Fitbit Charge HR), their willingness to engage with this type of technology and whether they would have any concerns with using these types of devices. Participants were also shown the companion applications from Withings and Fitbit and were asked about how they interpreted the data representations presented in both applications. The focus group was audio recorded and transcribed to allow for thematic analysis.

A round of semi-structured individual interviews were held with five older adults, 3F, age range 6876 , in order to gain insight into feeling and opinions related to mindfulness meditation and stress management in general. Participants were asked questions related to their prior experiences, if any, with meditation, their understanding of the concept, and also how interested they would be in trying it. In addition, they were asked to describe general stress levels, stressors and sources of stress or worry, and what techniques they employed to manage stress. The interviews were then transcribed for thematic analysis.

A second round of semi-structured individual interviews were held with six older informal carers, $5 \mathrm{~F}$, age range 53-74. Again, the interviews were semi-structured and covered a range of issues, notably the participants' experiences as carers, education and training related to caring, support received or lack thereof, and mechanisms for coping with stress. The participants were caring for relatives with co-morbidities, ranging in age from 75-92. The goal in this instance was to gain insight into how stress affects quality of life, health and wellbeing in general, and their capacity to provide care.

\subsection{Analysis}

Initial focus groups were analyzed qualitatively using thematic analysis in order to establish common structures and themes. Using semistructured interviews and focus groups allowed for insights into participants perceptions that would be difficult to obtain using other methods. Grounded Theory was then employed during the second round of interviews as an inductive research method to code the data as well as a theory generation method. While the requirements gathering phase gave rise to a theoretical framework that shaped the existing research questions, applying grounded theory to the posttrial interviews allowed further themes to emerge to generate new theory. NVivo, ${ }^{2}$ qualitative data analysis computer software, was used for analysis and enabled classification and sorting of information in order to examine relationships and identify trends and emergent themes.

http://www.qsrinternational.com/nvivo/nvivo-products

\section{FINDINGS}

\subsection{Focus Group 1}

The initial response to questions on general feelings towards health was one of optimism. Participants (P1 - P5) demonstrated a positive mental attitude towards health in general, discussing how they "go with the flow" and "live for today". This was motivated by a desire to remain independent, and not have to move to a nursing home. There was a consensus among the majority in this regard.

Emotional wellbeing and stress were touched on in relation to getting a good night's sleep with the idea of something being on your mind affecting sleep. P1 said: "You see, it depends what's on your mind when you go to bed", while P4 said: "That's the time you only get a few hours or unless there's somebody on your mind and your head's going, your brain's going". Health in general (both one's own or somebody close) can be a cause of stress. The idea of being helpless leads to constant worry and this preoccupation results in an inability to relax. P5 said, "I suppose health would be the biggest stress factor of most people". Thinking about ageing and mortality is also a cause of stress and anxiety.

The discussion showed that there are differing views on what constitutes sleep quality. An analysis with objective data from bed sensors would allow for a comparative analysis between sensor data and self-reported sleep quality and could reveal insights into the importance of feeling like you have had a good sleep. There was a feeling that a bad night's sleep will have a negative effect the following day.

Participants had different routines and also views on what will lead to a good night's sleep, for example eating, drinking water (and its effect on toilet use), reading, TV. There was a consensus that 6 hours plus is a good night's sleep for older people. The importance of having a routine when it comes to sleep was significant to all of the participants. P4 said: "Routine is a big thing. Going to bed at one o'clock one night, and going at 10, it's not working because if you get to bed at 10 o'clock, you're waking at one."

During the second part of the initial focus group, various visualisations of sensor-based data were shown to participants to gather their opinions on how intuitive each was and to understand how they might react to receiving such data. These included bar and line charts, various custom-made visualisations and visualisations from various selftracking applications such as Fitbit and Withings. Some issues with these interfaces and data visualisations included information overload and complex visualisations that were not easily 
interpretable by participants. There was a consensus that bar charts are the easiest form to understand. Participants expressed an interest in the idea of an objective sleep score, as was depicted on one of the visualisations. However, there was initial confusion as to what 'good' is in terms of percentages. P5 said: "And would that be good, 75\%? I suppose it would"

Two participants felt they could use sensor data to gain an insight into sleep trends and subsequently learn to alter habits and sleep better. P2 expressed an interest in knowing the reason behind a poor night's sleep. It is possible, therefore, that he could benefit from feedback via sensor data if he were to change behaviour accordingly. P5 also felt it would be "interesting to know what to do (with the data). P2 felt that the information in the graphs by itself isn't enough, suggesting it should be accompanied by suggestions or health tips. There was a positive reaction to the idea of educational messages and advice in general. P2 felt that more education and feedback would ultimately result in less dependence on medication. Participants agreed that finding the right balance is important, in terms of medication and lifestyle. There was also a feeling that it would be good to not have to rely on doctor visits so much, partly due to the fact that not enough time is afforded to patients when they visit a doctor.

Some of the main insights to emerge from this first focus group were:

- Overall positive attitude to health in generalis'

- Sense of independence is important to older adultsi'sépi

- Routine plays a role in wellbeing

- Stress and worry can affect sleepis

- Poor sleep will have a negative on the following day:

- Participants would be interested in useful feedback from sensor data

As a result, sensors were sought to monitor both stress and sleep. A number of sensors were under consideration including Emfit bed sensors (sleep), Withings blood pressure cuffs (stress), Fitbit Charge HR (sleep, activity, and heart-rate monitoring/stress), and Withings Activite Pop Watch (sleep and activity monitoring). Each of these devices offers different functionality and use cases, with varying degrees of precision. Based on feedback from older adults, the Withings Activite Pop (now Nokia Health Steel - Fig. 1) was selected and will be used to monitor steps and sleep. Another advantage to this device is its unobtrusiveness. It has an eight-month battery and does not require charging, and it can also be worn in the shower or while swimming. The Withings cuff (now Nokia Health) was selected to measure blood pressure. While this won't necessarily provide a fully accurate, objective measure of stress, stress has been linked to high blood pressure and it is therefore an important parameter of health to monitor in this application.

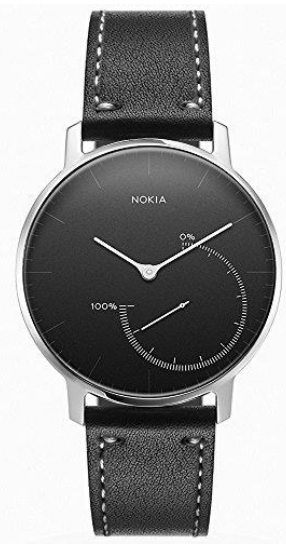

Figure 1: Nokia Health Steel

\subsection{Focus Group 2}

One of the most important factors raised by participants in Focus Group 2 (P6 - P9) in terms of growing older was retaining a sense of independence; this echoes sentiments expressed in Focus Group 1. Social interaction and staying active and how these issues can affect emotional wellbeing were both put forward as very important aspects of ageing, with P6 saying 'l'd get a bit depressed and feel miserable and think "oh l've got to get out"'. One participant saw keeping fit as dual purpose in that it results in both staying active and getting 'out and about' and remaining part of the community. While both P9 and P8 felt physical exercise and keeping active are key when growing older, they had differing views on the idea of goalsetting and targets, with P8 setting clear weekly targets to be achieved, and P9 not seeing much inherent value in doing this. As well as focusing on physical activity and being active members in the community, all of the participants agreed blood pressure was an important aspect of health for older adults to remain aware of.

In terms of sleep, P9 saw quality of sleep as more important than quantity for older adults. Sleep quality is seen as subjective by participants, with the idea being you will know you have had a good sleep by how you feel the following day. P9 said 'if you get a good sleep, you're going to feel it'. P7 pointed out that physical activity is important for maintaining good sleep hygiene, mentioning that having vertigo and not being as physically active as she had been in the past had caused her sleep quality to suffer. Participants differed in what part of the day they are most active. Two participants emphasized the importance of having a daily routine and pattern to your activities, suggesting an irregular or broken routine can in turn impact the 
ability to get a good night's sleep (P7: ...definitely, and if you break that pattern, you know the next day, you're not right - P6: Oh yeah). This was brought up during the first focus group also. Medical conditions can affect ability to perform physical tasks and activities and affect routine and pattern, which could in turn affect sleep.

During the second part of the focus group, participants were shown two wrist-worn activity trackers; Withings Activite Pop, and Fitbit Charge HR. Examples of data visualizations from both accompanying mobile applications were also displayed in order to ascertain how easy they were to interpret and to gain insight into participants' preferences for different means of data representation. These included charts and graphs displaying sleep and steps data with focus also being drawn to the use of various icons and symbols used in these applications,

The initial reaction to the Withings watch was positive, with participants finding it aesthetically pleasing and easy to read. P7 said 'Oh that's a very smart watch. It's nice and clear'. In terms of comfort, P9 found it to be comfortable and nonintrusive. The Fitbit device also received a positive response in terms of look and feel. The on-screen display was somewhat confusing at first but P7 understood once he was shown. It is possible that screen size on this device could be an issue for older adults, however. P8 saw the Fitbit Charge HR data as useful due to an existing heart condition. It should be noted that $\mathrm{P} 8$, however, had a negative reaction towards these devices due to fact that he does not like watches in general, saying 'I don't wear a watch so I don't like it'. As well as this, he has had negative experiences before with wearable medical devices, which he found bothersome and intrusive; this could result in low motivation to use another one (P8: ...I don't know why but it was constant, maybe I didn't know how to set it or whatever but it was constantly beeping). This theme was brought up by P9 also but for a different reason. It is possible his prior negative experiences with technology, in particular taking a PC course, could be affecting his motivation to using this type of device/app. However, it should be noted that a laptop was being used to display the screenshots from both mobile applications, which may have caused him to think it would be necessary to use a PC or laptop with these devices.

P7 was positive and somewhat curious about the devices overall but like P9 does not wear a watch in general. This could be an issue with users in terms of adherence and/or engagement. Indeed, P7 highlighted another possible issue with 'always on' monitoring devices. Though she indicated she wouldn't have a problem sleeping while wearing the device, she generally wouldn't wear a watch while performing household activities. The idea of a device being passive/in the background/nonintrusive appears to be important to this participant (11: That's waterproof. That one is sort of splash proof-P7: You see it would have to be).

In terms of interpreting and using data from these types of devices, contextual feedback supplemented with education and advice that can be acted upon was seen as important. It was also highlighted that feedback should not cause alarm or lead to unnecessary worry. P6 said 'you see l'd worry, "oh no there's something wrong there, I shouldn't have, I should get more"'. With regards to viewing sleep data, there was consensus that you know when you have had a good sleep, indicating that this cohort may not consider objective measures relevant. 'Gut feeling' was seen as more important than objective data. P8 said 'if I wake up in the morning, I know whether I feel good or I feel bad. I don't need to see 58 was my resting pulse. It's a subjective thing and I don't need that to tell me'. This is very relevant for self-report and how it compares to objective sensor data. P9 could perhaps benefit from actionable insights based on data if they contrast with subjective measures.

Three of the participants were already familiar with blood pressure readings. There was some confusion, however, over the relationship between $\mathrm{BP}$ and $\mathrm{HR}$, highlighting the need for training and education in this instance. Blood pressure was again brought up as an important parameter of health by the participants and is sometimes used as indicator of overall health ( $P 1-$ well I take my heart rate and blood pressure now once a week, P9 - I take mine every day). P7 did not see as much value in it as before, however. Two of the participants used journaling/logging as means to track changes and see trends in their blood pressure readings. Data was seen as more useful if it involves informing a doctor or when feeling ill as opposed to everyday feedback for self-reflection

\subsection{Interviews Round 1}

Following on from the two focus groups, a round of semi-structured interviews were held with five older adults $(\mathrm{P} 10-\mathrm{P} 14)$ in order to explore means of managing stress and feelings towards mindfulness meditation. Each of the first-round interview participants responded positively to the idea of mindfulness-based stressed reduction, with some more familiar with the concept than others. Having seen an introductory video about meditation in general, P10 was interested in finding out about the science behind the practice and also suggested ten minutes a day is practical and realistic. Instructions and background were seen as important by two participants, with one in particular suggesting education would be very important for motivation (P14: A reason why you're doing it -1 : Well the 
person has to believe in it as well - P14: Yeah, well you see you've to tell them why you're doing it).

The participants in general felt that stress reduction is a very important issue which can affect overall wellbeing and agreed that stress can have an adverse effect on sleep (P10: Well, I think something on your mind is certainly a factor). One participant already uses stress-reduction techniques, such as muscle relaxation, to help her get to sleep, which suggests this participant currently engages in health self-management techniques. Activity was seen as a very important element in getting a good night's sleep. In terms of stressors and issues which may cause older adults to worry in general, examples given included worrying about one's own health, retaining a sense of independence, and having to rely on a healthcare system. These sentiments were expressed in previous focus groups also. Different techniques are employed to handle feelings of stress, with one participant using distraction and acceptance (P11: I don't think it's of benefit to dwell on things like that. If you can do something about it, that's fine. If you can't do anything about it, it's pointless).

\subsection{Interviews Round 2}

The next round of semi-structured interviews was held with six informal carers (P15 - P20). The interviews explored the participants' experiences as carers, education and training related to caring, support received or lack thereof, and mechanisms for coping with stress. The interviews revealed a number of significant themes shared across each participant. An overarching theme throughout all of the interviews was carer burden and the sense of being overwhelmed, with comments such as 'It was just draining' and 'I couldn't cope with it anymore' (P20). P20 explained how the experiences of caring takes over her entire life, saying 'it does take over your life...he's dependent on me'. This idea of constantly having to be available and ready was also expressed by P19, saying 'you are always on duty'. When describing how caring affects her own life, P18 described a sense of chronic stress, differentiating between the stress of a deadline at work and the constant, overwhelming stress associated with caring. She said 'you just can feel...trapped sometimes so you kind of get a bit, oh I don't know, you just sort of, you get stressed just being there. You kind of want to scream'. Such feelings can in turn result in the carer harbouring feelings of resentment borne out of a sense of frustration and a perceived lack of gratitude (it's just the bit of resentment I suppose as well. I know I did offer to look after her and that but she expected it too ... sometimes, you feel a bit... that she doesn't recognise that I am married and because we don't have children she feels 'well if you don't have kids, what have you got to worry about?'- P18).
Referring to a consultant's comments that caregiver burden is an overused and inappropriate term, P16 said 'I found that really offensive that a professional would actually say that'. The area of support was also mentioned throughout the interviews. P18 said 'I don't have kids...and I am more in a position to do it - it's just at times, it gets to you, you know'. The lack of support and the impact this can have in terms of loneliness was expressed by P20, saying 'it's hard, being at home and not having someone to lean on'. This was echoed by P19, saying 'There is no-one caring for the carer' and then later 'You feel vulnerable'. The sense of loneliness and social isolation was evident in comments such as 'Saturdays and Sundays you kind of feel everybody is doing things...weekends I find harder' (P18). And also 'I want to be out and about and it isn't working...I miss the socialising'. This sentiment was echoed by P20. Describing the fact that the recipient's mobility means he is unable to leave the house, she said 'it's like a prison sentence'. P19 said 'You put yourself last... You put them first'. Such comments suggest informal carers see caring as having a significant impact on their and emotional wellbeing and quality of life. Experiences of loneliness, social isolation, and reduced quality of life were also described by participants in Vaseliou et al. (2017)'s study investigating issues associated with being an informal caregiver.

One word that came up regularly throughout each of the interviews was 'challenge'. When asked about the challenges she faces, P20 said that one of her main challenges is not knowing what to expect from day to day, saying 'Fear, I have to say fear'. Describing the experiences of finding her husband having a stroke and how this would then affect their quality of life, P20 said 'I found it very daunting...it was just awful... livelihood gone in a flash...and he worked very hard'. Quality of life in terms of family relationships was also discussed, with $\mathrm{P} 16$ saying 'it's very demanding and it takes a toll on all of your relationships', and later 'so that's a huge challenge as well trying to keep that balance in place'. P18 also discussed this and explained how living with the recipient eventually took its toll on her relationship, saying 'so we did stay for a couple of months, but we just found it was impeding our relationship as well'.

Seeing the effects of conditions on the recipient affects the carer and can affect emotional wellbeing, particularly when there is such a close relationship. P16 said 'she is emotionally or psychologically challenged by her dementia, she is physically challenged by her heart... she would get breathless so you know that she is not in a great place...so you are kind of thinking ok...so yeah that's the challenge'. The sense of constant worry was a theme that also emerged from the interviews. P16 said 'your time isn't really your own well at this level as it gets harder. It is very hard 
because if you are not physically there, you are still worrying'. Such future-oriented worry could be addressed by the present moment focus of mindfulness in mindfulness-based cognitive therapy (Helmes et al. 2015). The sense of guilt arising from trying to balance caring with family commitments, resulting in being away from the care recipients was touched on ('I find that hard...I don't want to hurt her feelings' and also 'you are kind of feeling guilty because you don't think, don't want to be in the house' P18). Such experiences were revealed in Chen et al. (2015)'s findings also. The responsibility that accompanies caring and the extent to which the recipient depends on the carer can also lead to feelings of guilt, as expressed by P18 'she likes to know you are there...you feel guilty constantly'.

Some of the main insights and themes to emerge from these interviews were:

- Chronic stress more than acute stress

- Heath service support (and lack thereof)

- Family support (and lack thereof)

- Daily challenges

- Constant sense of worrying

- Carer burden

- Carer tasks can seem endless

- Care recipient's perceptions sometimes overlooked

- Feeling overwhelmed

- Loneliness

- Resentment

- Sense of guilt

- Stressors/Triggers

- Quality of life

- Emotional wellbeing and the impact of caring

\section{DISCUSSION AND APPLICATION DESIGN}

Insights gathered from the requirements gathering and testing sessions were vital in gaining an understanding of parameters of health which are important to older adults, feelings towards wearable devices and monitoring, preferences for data visualizations, carers' experiences, and attitudes to mindfulness as a means of stress reduction. Qualitative feedback from the focus groups and interviews indicated that stress management and sleep hygiene are areas that affect older adults' and informal carers' wellbeing, and persuasive technology could allow for behaviour change interventions to address these issues.

This research aims to address gaps in the literature concerning the area of mobile device/computermediated stress reduction interventions for older informal carers using mindfulness-based interventions and wearable activity trackers. Taking considerations from the requirements gathering findings into account, a high fidelity mock- up was designed, using Sketch for $\mathrm{Mac}^{3}$, which included four parameters of health: stress reduction/mindfulness, sleep, steps/activity and blood pressure. The application has 3 main elements: objective data input (collected via the wrist-worn sensor for sleep and steps and via the blood pressure cuff for blood pressure), subjective data input (self-report and annotation will allow for the collection of subjective measures of wellbeing, including stress), feedback / output (educational tips and advice messages based on goals achieved will be provided also). Finally, mindfulness-based intervention material and techniques will be provided on a daily basis through the 'Mind' section of the application.

As this application will display data from multiple sources, each with its own existing interface, the challenge will be to design a new interface which aggregates these data sources and presents them in a manner which is easily understood by an older user. Moreover, it will be necessary to consult with the user throughout the design cycle to establish which areas of functionality currently offered by these sensor technologies the users feel are of benefit to them (Nunes et al. 2015) This is particularly important with this user profile as there could be a risk of 'feature saturation', which might result in unnecessary user burden, or lack of interest. Doyle et al. (2014) point out that complex interfaces can make it difficult for older adults to benefit from health self-management technologies [3]. [is:e?

\subsection{Features and Functionality}

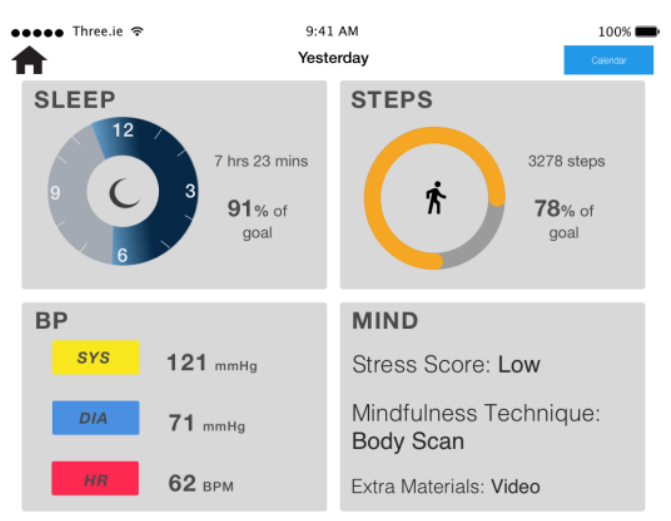

Figure 2: Home/Dashboard

The dashboard (Fig. 2) allows for a quick overview of all recent sensor data and self-reported stress scores. The user can click on any of these tiles to view more detailed information including trends over time, view feedback and the mindfulness interventions.

\footnotetext{
${ }^{3}$ https://www.sketchapp.com
} 


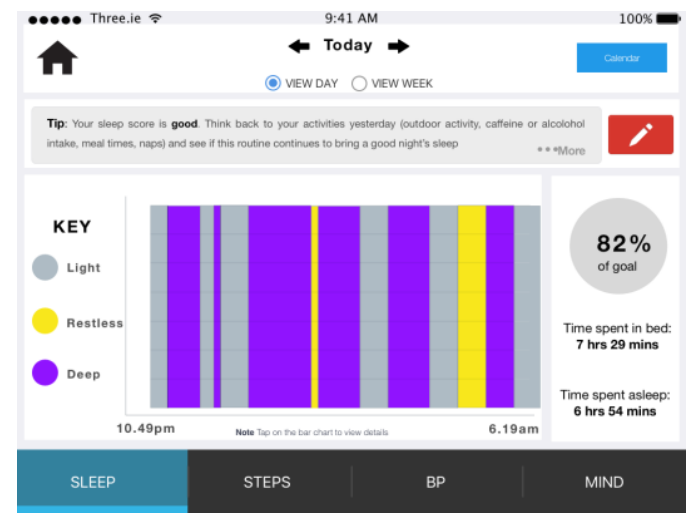

Figure 3: Sleep-Day View

Participants expressed an interest in useful feedback from sensor data such as sleep (Fig. 3). The Day view provides feedback on the previous night's sleep using a bar chart, with a breakdown of sleep states throughout the night. An educational tip is also provided based on the percentage of goal reached. Users can also annotate to provide further contextual information, which can be used for review and for self- reflection. Users can view previous days using the arrow at the top centre of the screen. The house icon at the top left of the screen will bring the user back to the dashboard. The overlay menu at the bottom of the screen is used for navigation throughout the four main sections of the application and also provides a sense of location within the application.

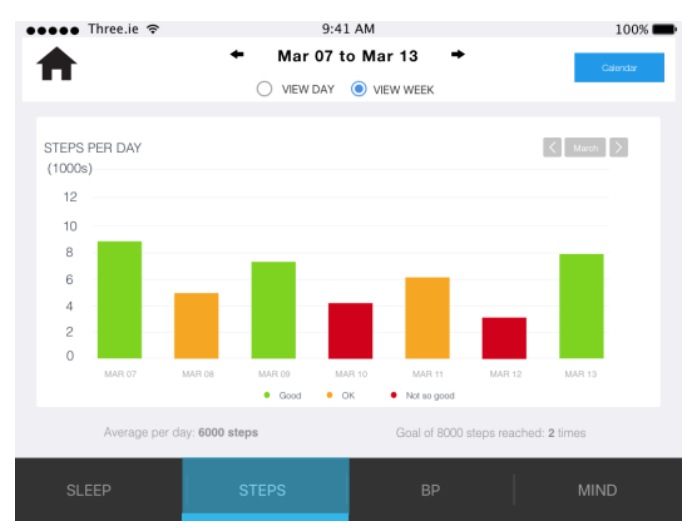

Figure 4: Steps - Week View

Activity was seen by participants as a very important contributor to getting a good night's sleep (Fig. 4). Using the toggle at the top centre of the screen, users can also view their data for the last week. This functionality is provided for each of the four sections of the app and allows for an overview of different levels reached throughout the week. Such an overview allows the user to "compare different values, to become aware about their health status, and to even find patterns to test and adjust their self-management' (Nunes et al. 2015). Users can navigate from week to week for further analysis using the left and right arrows above the toggle.

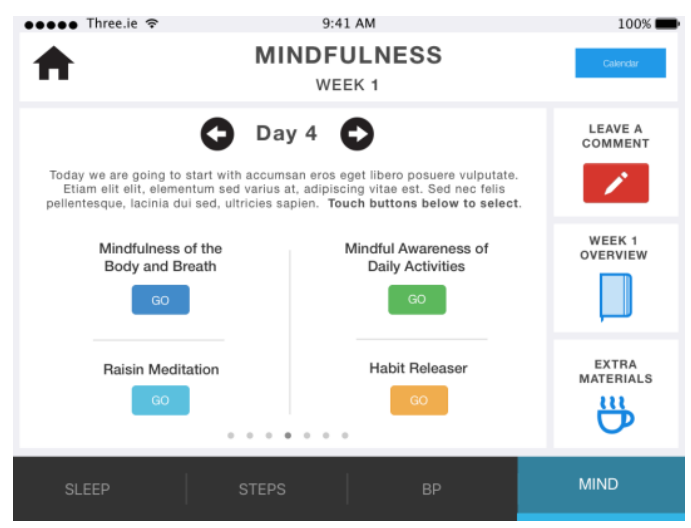

Figure 5: Mind - Day View

Mindfulness meditation content (Fig. 5) will be provided through the Mind section of the application. Each day the application will present the user with that day's practices, both in audio and text format.

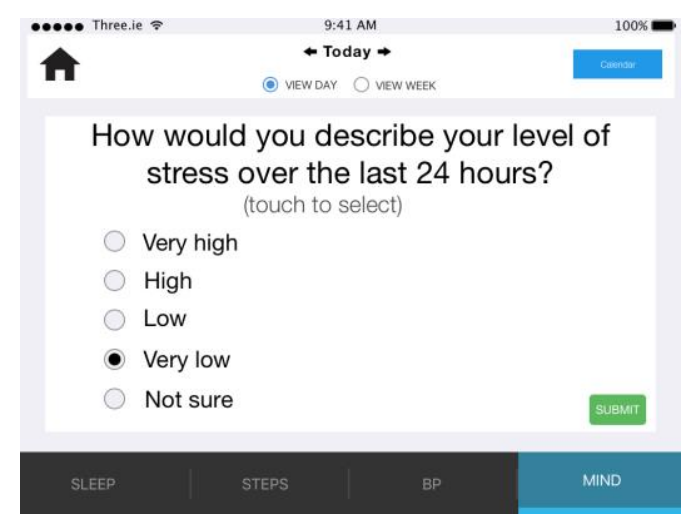

Figure 6: Mind - Self-report

Users will self-report each day using multiplechoice questions (Fig. 6). Wahbeh et al (2016) see this as the most effective means of self-reporting on a touch screen for older adults.

\subsection{Mindfulness Content}

The application will deliver guided mindfulness meditation daily both in audio and text format. The content has been sourced from the book 'Mindfulness - a practical guide to finding peace in a frantic world' and is used with permission of the authors Mark Williams and Danny Penman (2011). This book provides a guided 8-week course of mindfulness practice. It is particularly useful for this cohort as it does not require group sessions or day retreats as commonly found in most full $\mathrm{MBI}$ courses. Rather, a series of simple practices, which can be incorporated into daily life, are provided. The goal in this instance is to educate the user such that they will arrive at a point wherein 
mindfulness awareness practice can be deployed and utilized at will in times of high stress or anxiety (Visser et al. 2015).

Topics and practices covered include Habit Releaser, Mindful awareness of a routine daily activity, Mindfulness of the Body and Breath, Body Scan, Mindful Movement meditation, Three-minute Breathing Space meditation, Sounds and Thoughts meditation, Exploring Difficulty meditation, Befriending meditation (Fig. 4). The content provided in the application will aim to address issues raised by carers during the semi-structured interviews, such as constant worrying and negative thoughts. Each day the application will present the user with that day's practices, both in audio and text format. As they move through the eight-week course, they will be encouraged to incorporate these new skills and practices into their everyday lives. Mindfulness skills aim to help participants to accept negative thought patterns and to address these in an intentional and skilful manner (Fresco et al. 2010). Such skills should therefore empower informal carers to manage chronic stress associated with caring as reported in the interviews. Mindfulness could also lead to an improvement in sleep quality and efficiency resulting in turn to an enhanced sense of physical and mental energy (Visser at al. 2017). Improvements in anxiety, loneliness, and stress have been demonstrated as a result of online mindfulness interventions for older informal carers (Tkatch et al. 2017). The pilot study was unique in that it required less time and lower intensity and allowed participants to log on and practice from their homes. This project aims to build on the results seen in Tkatch et al. (2017)'s study by integrating sleep and activity data and feedback from a wrist-worn sensor and providing mindfulness interventions through a dedicated mobile application.

\section{CONCLUSION AND FUTURE WORK}

In this paper, we presented findings from a qualitative study with both older adults and older informal carers. Findings indicated that stress and sleep management were found to be particular areas of concern and can affect older adults' and informal carers' wellbeing. Participants viewed stress as having an adverse effect on sleep quality. An underlying and significant theme throughout all of the interviews was carer burden and the sense of being overwhelmed. The sense of constant and often future-oriented worry was also a notable issue to emerge from the interviews. Persuasive technology could allow for behaviour change interventions to address these issues. Mindfulnessbased interventions (MBIs) have grown in popularity in recent years and have been shown to reduce stress and increase quality of life among older informal carers. A digital application delivering MBIs has been designed which aims to support older informal carers to manage stress and sleep. The application design will be tested with informal carers in order to gauge usability and effectiveness in the following areas: interaction, size and spacing, ease of use, functionality, engagement and motivation, look and feel, navigation. This will then allow for a final design iteration to be developed prior to the main study. This research aims to address gaps in the literature concerning the area of mobile device/computermediated stress reduction interventions for older informal carers using mindfulness-based interventions and wearable activity trackers

A 12-week home-based study will be conducted to evaluate usage of the application with 15 older informal carers. Participants will self-monitor using a combination of sensing and self-report and will receive information and education feedback and a mindfulness intervention. Data to be collected will include: sensor data (captured daily), self-report data (captured daily), questionnaire data on stress, sleep and quality of life (pre- and post-trial), and semi-structured interview data (end of trial).

The system is designed to allow older informal carers to manage stress through mindfulnessbased interventions as well as monitor and manage their activity and sleep patterns through tracking, data visualizations and educational advice. The primary focus will be to understand how and why participants use the application. This will happen through an exploration of issues around usage, usefulness, acceptance, motivation and user experience. Secondary outcomes will involve examining any reductions in stress and improvements in sleep. This is in line with current thinking on how $\mathrm{HCl}$ technologies to support health/wellbeing should be evaluated - given that such technologies are typically early stage prototypes it cannot be expected that they will bring about the expected change in wellbeing (Klasnja et al. 2011).

Following data analysis, recommendations will be made for best-practice in designing applications that effectively support older informal carers in wellbeing self-management, as well as the potential of digital MBIs and wellbeing feedback to support this, which we believe will represent a significant contribution to the area of designing such applications for this cohort. Furthermore, this research could lay the foundation for further advancements in the area of stress management for older informal carers with the ultimate goal of reducing stress and improving sleep through a combination of self-reflection, education and mindfulness-meditation techniques, leading to an overall improvement in wellbeing and quality of life. 


\section{REFERENCES}

Bardram, J. E., Frost, M., Szántó, K., FaurholtJepsen, M., Vinberg, M., \& Kessing, L. (2013). Designing mobile health technology for bipolar disorder: a field trial of the monarca system. Proceedings of $\mathrm{CHI} 2013,2627-2636$. https://doi.org/10.1145/2470654.2481364

Bulmer, M. (1992) The Social Basis of Community Care. In Bornat, J. Community Care: A Reader. Open University DOI 10.1007/978-1-349-260874_5

Vasileiou, K., Barnett, J., Barreto, M., Vines, J., Atkinson, M., Lawson, S., \& Wilson, M. (2017). Experiences of loneliness associated with being an informal caregiver: $A$ qualitative investigation. Frontiers in Psychology, 8(APR). https://doi.org/10.3389/fpsyg.2017.00585

Carers UK (2009) Policy Briefing: Facts about Carers.

www.carersuk.org/Professionals/ResourcesandB riefings/PolicybriefingsFactsaboutcarersJun e2009.pdf

Kamiya, Y., Murphy, C., Savva, G. and Timonen, V. (2012) Profile Of Community- Dwelling Older People With Disability And Their Caregivers In Ireland http://tilda.tcd.ie/assets/pdf/Carer\%20Report.pdf

Brummett, B. H., Babyak, M. A., Siegler, I. C., Vitaliano, P. P., Ballard, E. L., Gwyther, L. P., \& Williams, R. B. (2006). Associations among perceptions of social support, negative affect, and quality of sleep in caregivers and noncaregivers. Health Psychology, 25(2). https://doi.org/10.1037/0278-6133.25.2.220

Care Alliance. (2012). Family Caring in Ireland [online]. Available from: is: \%202013\%20FA\%20130513.pdf

Chen, Y., Ngo, V., \& Young, P. S. (2013). Caring for Caregivers: Designing for Integrality. In Proceedings of the 2013 conference on Computer supported cooperative work (Vol. 91102, pp. 18-20). https://doi.org/10.1145/2441776.2441789

Montgomery, R. J. V., \& Kosloski, K. (2009). Caregiving as a Process of Changing Identity: Implications for Caregiver Support. Generations, 33(1), 47-52.

Adams, P., Rabbi, M., Rahman, T., Matthews, M., Voida, A., Gay, G., Choudhury, T., sięVida, S. (2014). Towards personal stress informatics: comparing minimally invasive techniques for measuring stress in the wild. In Pervasive Health, 2014, pp. 72-79. DOI: http://eudl.eu/doi/10.4108/icst.pervasivehealth.2 014.254959

Bohlmeijer, E., Prenger, R., Taal, E., \& Cuijpers, P. (2010). The effects of mindfulness- based stress reduction therapy on mental health of adults with a chronic medical disease: a meta-analysis. Journal of Psychosomatic Research, 68, 539544. SEE:

Bauer, J.S., Consolvo, S., Greenstein, B., Schooler, J., Wu, E., Watson, N.F., Kientz, J. 2011. ShutEye: Encouraging awareness of healthy sleep awareness with a mobile, peripheral display. In $\mathrm{CHI} 2012$, pp. 1401-1410 http://dl.acm.org/citation.cfm?doid=2207676.220 8600

Black, D.S., O'Reilly, G.A., Olmstead, R., Breen, E.C., Irwin, M.R. (2015). Mindfulness Meditation and Improvement in Sleep Quality and Daytime Impairment Among Older Adults With Sleep Disturbances: A Randomized Clinical Trial. In JAMA Internal Medicine 175(4) SLEE?

Chiesa, A., \& Serretti, A. (2011). Mindfulnessbased interventions for chronic pain: a serfystematic review of the evidence. The Journal of Alternative and Complementary Medicine, 17, 83-93. "ispe?

Dharmawardene, M., Givens, J., Wachholtz, A., Makowski, S., \& Tjia, J. (2016). A systematic review and meta- analysis of meditative interventions for informal caregivers and health professionals. BMJ Supportive \& Palliative Care, 6 , 160-169. https://doi.org/10.1136/bmispcare-2014-000819

Wahbeh, H., Goodrich, E., Oken, B.S. Internetbased Mindfulness Meditation for Cognition and Mood in Older Adults: A Pilot Study. Alternative Therapies 2016 Mar;22(2):44-53

Fresco, D.M., Flynn, J.J., Mennin, D., Haigh, E.A.P., (2010) Mindfulness Based Cognitive Therapy In J. D. Herbert, E. Forman (eds): Acceptance and Mindfulness in Cognitive Behavior Therapy. Wiley.

Mallya, S., \& Fiocco, A. J. (2016). Effects of Mindfulness Training on Cognition and WellBeing in Healthy Older Adults. Mindfulness, 7(2), 453-465. https://doi.org/10.1007/s12671-0150468-6

Dutton, G.R. (2008), The Role of Mindfulness in Health Behavior Change. ACSM s Health \& Fitness Journal 12(4):7-12 DOI 10.1249/FIT.0b013e31817bf5db

Kristeller, J. L., Baer, R. A., \& Quillian-Wolever, R. (2006). Mindfulness-Based Approaches to Eating Disorders. In Mindfulness-Based Treatment Approaches (pp. 75-91). Elsevier Inc. 
https://doi.org/10.1016/B978-012088519$\underline{0 / 50005-8}$

Tkatch, R., Bazarko, D., Musich, S., Wu, L., MacLeod, S., Keown, K., ... Wicker, E. (2017). A Pilot Online Mindfulness Intervention to Decrease Caregiver Burden and Improve Psychological Well-Being. Journal of EvidenceBased Complementary and Alternative Medicine, 22(4), $736-743$. https://doi.org/10.1177/2156587217737204

Helmes, E., \& Ward, B. G. (2017). Mindfulnessbased cognitive therapy for anxiety symptoms in older adults in residential care. Aging \& Mental Health, 21(3), 272-278. https://doi.org/10.1080/13607863.2015.1111862

Nunes, F., Verdezoto, N., Fitzpatrick, G., Kyng, M., Grönvall, E., \& Storni, C. (2015). Self-Care Technologies in $\mathrm{HCl}$. ACM Transactions on Computer-Human Interaction, 22(6), 1-45. https://doi.org/10.1145/2803173

Doyle, J., Walsh, L., Sassu, A., McDonagh, T. 2014. Designing a Wellness Self- management tool for older adults - results from a field trial of YourWellness.. In Proceedings of the $8^{\text {th }}$ International Conference on Pervasive Computing Technologies for Healthcare (Oldenburg, Germany May 20-23, 2014) Pervasive Health, 134-141. is.pi

Williams, M., \& Penman, D. (2011). Mindfulness: a practical guide to finding peace in a frantic world. Hachette UK.

Visser, P. L., Hirsch, J. K., Brown, K. W., Ryan, R., \& Moynihan, J. A. (2015). Components of Sleep Quality as Mediators of the Relation Between Mindfulness and Subjective Vitality Among Older Adults. Mindfulness, 6(4), 723-731. https://doi.org/10.1007/s12671-014-0311-5

Klasnja, P., Consolvo, S. and Pratt, W. How to evaluate technologies for health behaviour change research. In $\mathrm{CHI}$ '11, 2011. ispe: 